EPJ Web of Conferences 21, 07003 (2012)

DOI: $10.1051 /$ epjconf/20122107003

(C) Owned by the authors, published by EDP Sciences, 2012

\title{
Deuteron-induced reaction mechanisms at low energies
}

\author{
M. Avrigeanu, a, V. Avrigeanu, and F.L. Roman \\ "Horia Hulubei" National Institute for Physics and Nuclear Engineering, P.O. Box MG-6, 76900 \\ Bucharest, Romania
}

\begin{abstract}
An extended analysis of the reaction mechanisms involved within deuteron interaction with nuclei is presented. An increased attention is devoted to the breakup mechanism, all its components, namely the elastic, inelastic (fusion), and total breakup being carefully considered. Next, the direct reactions represented by the stripping and pick-up mechanisms are discussed. Finally, the pre-equilibrium and compound-nucleus contributions, corrected for the breakup, stripping, and pick-up decrease of the total reaction cross section, complete the deuteron-induced reaction cross section analysis. The overall agreement between the measured data and model calculations validates the description of nuclear mechanisms taken into account for the deuteron-nucleus interaction.
\end{abstract}

\section{Introduction}

The deuteron-induced reactions at low and medium energies have a great importance within several on-going strategic research programmes for international large-scale facilities as ITER (International Thermonuclear Experimental Reactor), IFMIF (International Fusion Material Irradiation Facility), and SPIRAL-2 (Système de Production d'Ions Radioactifs en Ligne - generation 2). Particularly, the Neutron For Science (NFS) project at SPIRAL2, that involves deuteron beams, is focused on both academic research and applied physics related to both neutron- and deuteron-induced reactions. The accurate knowledge of the deuteron activation cross sections is critical for selecting and validating the best structural materials and a number of key technologies, while the actual data of deuteron-induced reaction are less extensive and mature than for neutrons. Consequently, there is now a large attention paid to further measurements as well as to improved model calculations becoming finally able to describe the experimental data in order the deuteron data libraries to approach the standard of the established neutron ones.

The description of deuteron-nucleus interaction represents an important test for both the appropriateness of reaction mechanism models and evaluation of nuclear data requested especially by the above mentioned research programmes. The weak binding energy of the deuteron, $\mathrm{B}=2.224 \mathrm{MeV}$, is responsible for the high complexity of the interaction process that supplementary involves a variety of reactions initiated by the neutrons and protons following the deuteron breakup. This has been the reason hampering so far the comprehensive analysis involving large A-range of targets and incidentenergy domain. The difficulties to interpret the deuteron-induced reaction data in terms of the usual reaction mechanism models have recently been re-investigated [1-8] looking for a consistent way to include the breakup contribution within the activation cross section calculations too.

On the other hand, usually neglected or very poorly taken into account, the (d,p) and (d,n) stripping as well as the $(\mathrm{d}, \mathrm{t})$ pick-up reaction contributions have been shown to be important at low incident energies [1-8], requesting an appropriate treatment within, e.g., the Coupled-Reaction Channels (CRC) formalism. In this respect, the present work concerns a deeper understanding of deuteron breakup, stripping and pick-up reactions, all together and consistently with the better-known and described statistical emission.

\footnotetext{
a e-mail: Marilena.Avrigeanu@nipne.ro
}

This is an Open Access article distributed under the terms of the Creative Commons Attribution-Noncommercial License 3.0, which permits unrestricted use, distribution, and reproduction in any noncommercial medium, provided the original work is properly cited. 


\section{EPJ Web of Conferences}

\section{Deuteron breakup effects on activation cross sections}

The physical picture of the deuteron-breakup in the Coulomb and nuclear fields of the target nucleus considers two distinct chains, namely the elastic-breakup (EB) in which the target nucleus remains in its ground state and none of the deuteron constituents interacts with it, and the inelastic-breakup or breakup fusion $(\mathrm{BF})$, where one of these deuteron constituents interacts with the target nucleus while the remaining one is detected.

An empirical parametrization of the total proton-emission breakup fraction from the deuteron total reaction cross section $\sigma_{R}, f_{B U}^{(p)}=\sigma_{B U}^{p} / \sigma_{R}$, and elastic breakup fraction, $f_{E B}=\sigma_{E B} / \sigma_{R}$, have been obtained [1] from the analysis of the experimental systematics [9-13] of the proton-emission spectra and angular distributions of deuteron-induced reactions on target nuclei from $\mathrm{Al}$ to $\mathrm{Pb}$, at incident energies from 15 to $80 \mathrm{MeV}$. Their dependence with respect to the charge $\mathrm{Z}$ and atomic number of the target nucleus A, as well as deuteron incident energy E is [1]:

$$
\begin{array}{r}
f_{B U}^{(p)}=0.087-0.0066 Z+0.00163 Z A^{1 / 3}+ \\
0.0017 A^{1 / 3} E-0.000002 Z E^{2}, \\
f_{E B}=0.031-0.0028 Z+0.00051 Z A^{1 / 3}+ \\
0.0005 A^{1 / 3} E-0.000001 Z E^{2} .
\end{array}
$$

A comparison with the total proton- and neutron-emission breakup cross-section parametrization of Kalbach [14] :

$$
\sigma_{B U}^{b}=K_{d, b} \frac{\left(A^{1 / 3}+0.8\right)^{2}}{1+\exp \frac{(13-E)}{6}}, \quad K_{d, p}=21, \quad K_{d, n}=18 .
$$

shows that the former parametrization [1] considers equal breakup fractions for proton and neutron emission, but supplementary gives all breakup components, i.e. the total, elastic, and inelastic fraction $f_{B F}^{b}=f_{B U}^{b}-f_{E B}$. It can be seen in Fig. 1(a) that both parameterizations predictions for ${ }^{63,65} \mathrm{Cu}$, ${ }^{59} \mathrm{Co}$, and ${ }^{93} \mathrm{Nb}$ target nuclei are close for deuteron incident energies above $\sim 7 \mathrm{MeV}$. However, the total protonemission breakup cross sections given by Kalbach parametrization [14] remain higher than even the deuteron total reaction cross sections $\sigma_{R}$ with the incident energy decrease (Fig. 1).

\subsection{Phenomenological EB versus CDCC formalism}

Concerning the energy dependence of the inelastic- and elastic-breakup components, the interest of the deuteron activation for incident energies up to $30 \mathrm{MeV}$ has motivated an additional check [15] of the elastic-breakup parameterization extension beyond the energies formerly considered for the derivation of its actual form.

Actually, our parameterization [1] for the elastic-breakup was obtained from the analysis of the empirical systematics which covers an incident energy range from 15 to only $30 \mathrm{MeV}$. However, as it is shown in Fig. 1(b) for the ${ }^{63} \mathrm{Cu}$ and ${ }^{93} \mathrm{Nb}$ target nuclei, the elastic-breakup cross sections given by the empirical parameterization [1] decrease with the incident energy beyond the energy range within which it was established, while the total-breakup cross section has an opposite trend. Therefore, in the absence of available experimental deuteron elastic-breakup data at incident energies above $30 \mathrm{MeV}$, the correctness of an eventual extrapolation should be checked by comparison of the related predictions with results of an advanced theory as, e.g., the Continuum-Discretized Coupled-Channels (CDCC) method [16-21].

The elastic-breakup component is treated within the CDCC formalism as an inelastic excitation of the projectile due to the nuclear and Coulomb interactions with the target nucleus. Consideration of this excitation is performed through the coupling of the projectile unbound excited states in the solution of the scattering problem by means of the coupled channels approach. The deuteron scattering is analyzed within a three-body model, comprising the two-body excited projectile and the inert target nucleus. The 

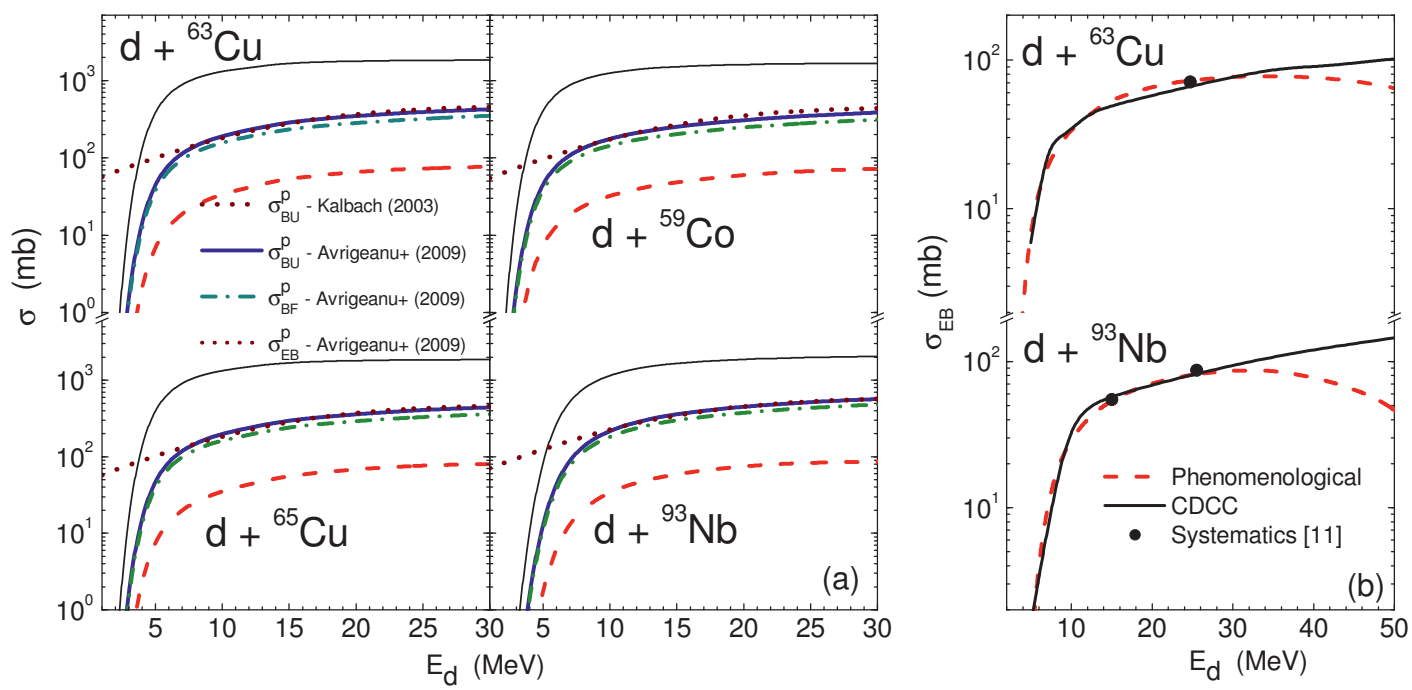

Fig. 1. (Color online) (a) The energy dependence of the total (thick solid curves), elastic (dashed), and inelastic (dot-dashed) proton-emission breakup cross sections [1] and total proton-emission breakup cross sections [14] (dotted) for deuteron interactions with ${ }^{63,65} \mathrm{Cu},{ }^{59} \mathrm{Co}$, and ${ }^{93} \mathrm{Nb}$, while the deuteron total reaction cross section $\sigma_{R}$ is shown by thin curves. (b) Energy dependence of the empirical [1] (dashed curve) and CDCC [15] (solid) elastic breakup cross sections for deuteron scattering on ${ }^{63} \mathrm{Cu}$ and ${ }^{93} \mathrm{Nb}$ target nuclei. The solid circle is the value from Kleinfeller systematics [11].

energy dependence of the elastic-breakup cross sections provided by the excitation of the continuum spectrum, in the case of the deuteron interaction with ${ }^{63} \mathrm{Cu}$ and ${ }^{93} \mathrm{Nb}$ target nuclei, is compared with the prediction of empirical systematics [1] in Fig. 1(b). The elastic-breakup cross sections corresponding to the Kleinfeller et el. systematics (Table 3 of Ref. [11]) are also shown. The agreement of the CDCC elastic-breakup cross sections [15] and the latter systematics can be considered as a validation of the present advanced model approach. Moreover, the comparison shown in Fig. 1(b) points out that the CDCC calculations lead to elastic-breakup cross sections which follow the total-breakup cross section behavior as well as that of the reaction cross section shown in Fig. 1(a), and makes clear that the empirical parameterization extrapolation for the elastic-breakup cross sections beyond the energies considered in this respect should be done with caution [15].

The CDCC method thus provides useful initial guidance for the assessment of these extrapolation accuracies and may help to improve the existing phenomenological approaches. However, additional experimental deuteron interaction data, like elastic-scattering angular distributions and inclusive neutron and proton spectra, are needed in order to validate the parameters involved in the CDCC as well as to complete the systematics of the elastic- and total-breakup cross sections over enlarged energy and target mass domains.

\subsection{Inelastic-breakup enhancement of the deuteron activation cross sections}

On the whole, the breakup process reduces the amount of the total reaction cross section that should be shared among different outgoing channels. On the other hand, the inelastic-breakup component, where one of deuteron constituents interacts with the target leading to a secondary composite nucleus, bring contributions to different reaction channels. The secondary-chance emission of particles from the original deuteron-target interaction is thus especially enhanced. Therefore, the absorbed proton or neutron following the breakup emission of a neutron or proton, respectively, contributes to the enhancement of the corresponding $(d, x n)$ or $(d, x p)$ reaction cross sections. In order to calculate this breakup enhancement for, e.g., the $(d, x n)$ reaction cross sections, firstly the inelastic-breakup cross sections were obtained by subtracting the elastic-breakup cross sections from the phenomenological 

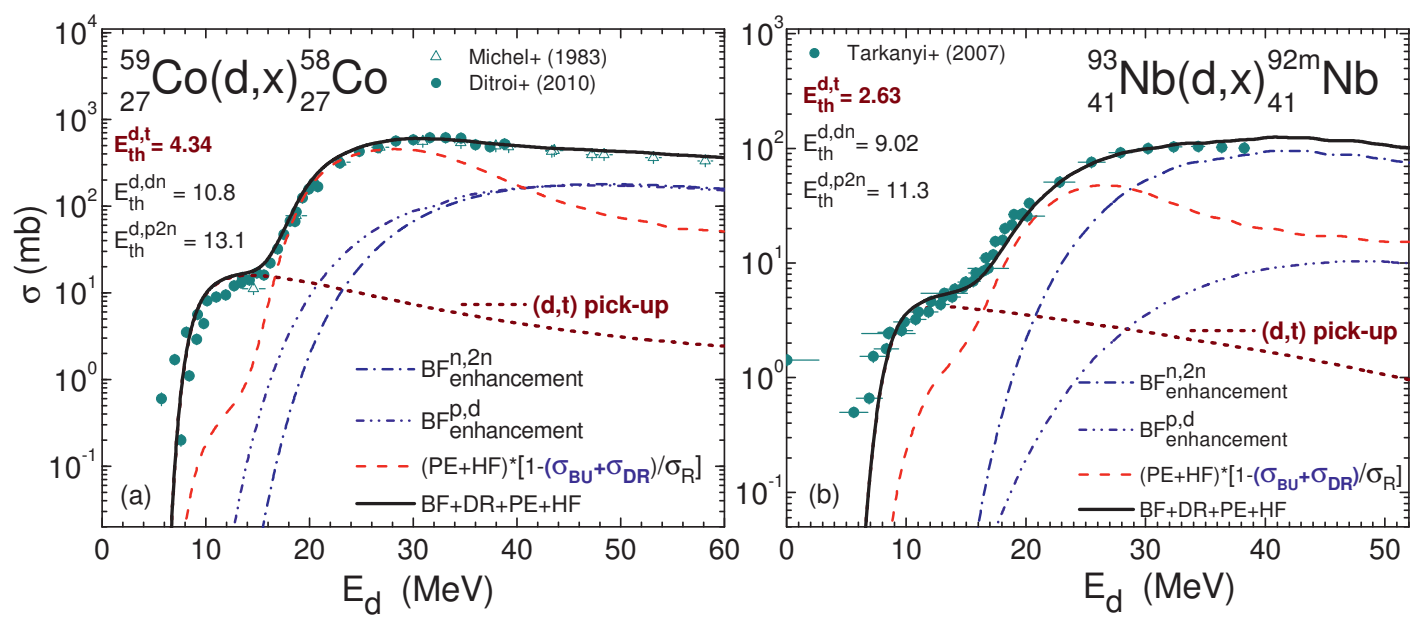

Fig. 2. (Color online) Comparison of measured data [23] for deuteron interactions with the ${ }^{59} \mathrm{Co}$ (left) and ${ }^{93} \mathrm{Nb}$ (right) target nuclei, and complete analysis results (solid curves) taking into account the deuteron inelastic breakup enhancement through $(n, 2 n)$ (dot-dashed) and $(p, d)$ (dot-dot-dashed) reactions, the $(d, t)$ pick-up reaction (dotted), and the $\mathrm{PE}+\mathrm{CN}$ (dashed) contributions to the activation of ${ }^{58} \mathrm{Co}$ and ${ }^{92 m} \mathrm{Nb}$ residual nuclei.

total nucleon-breakup cross sections. Next, they have been multiplied by the ratios $\sigma_{(p, x)} / \sigma_{R}$ corresponding to the above-mentioned reactions of the absorbed proton with the target nucleus, where $\sigma_{R}$ is the proton reaction cross section and $x$ stands for various e.g. $\gamma, n, d$, or $\alpha$ outgoing channels [27]. These ratios have been expressed as function of the deuteron incident energy using the Kalbach [22] formula for the peak energies of the deuteron-breakup emitted constituents in the center-of-mass system:

$$
\epsilon_{n(p)}=\frac{1}{2}\left(\epsilon_{d}-B_{d} \mp \frac{1.44 Z}{1.5 A^{1 / 3}+3.1}\right) .
$$

The inelastic-breakup contributions to the $(d, x p)$ activation cross section due to the neutrons absorbed in further interactions with the target nucleus have been obtained in a similar way. The only difference was the replacement of the ratios $\sigma_{(p, x)} / \sigma_{R}$ by the ratios $\sigma_{(n, x)} / \sigma_{n o n}$, where the non-elastic cross section $\sigma_{\text {non }}$ plays the same role for neutrons as $\sigma_{R}$ for protons.

Since the assumed Gaussian line shape of the deuteron-breakup peak energies of the emitted constituents have quite large widths, a better estimation of inelastic breakup enhancement consists in a convolution of either the ratio $\sigma_{(n, x)} / \sigma_{n o n}$ or $\sigma_{(p, x)} / \sigma_{R}$ with the Gaussian line shape of the deuteronbreakup peak energies of the corresponding emitted constituent [6]. The resulting inelastic breakup contributions corresponding to $(n, 2 n)$ and $(p, d)$ processes on ${ }^{59} \mathrm{Co}$ and ${ }^{93} \mathrm{Nb}$ target nuclei calculated through the convolution procedure are shown in Fig. 2.

\section{One-nucleon transfer reactions}

Apart from the breakup contributions to deuteron interactions, an increased attention has to be devoted to the direct reactions very poorly accounted so far in deuteron activation analysis. For deuteron energies below and around the Coulomb barrier the interaction of deuterons with target nuclei proceeds largely through direct reaction (DR) mechanism, while processes like pre-equilibrium-emission (PE) or evaporation from fully equilibrated compound nucleus $(\mathrm{CN})$ become also important with the increase of the incident energy.

The appropriate consideration of the DR mechanisms, like stripping and pick-up, that are important at the low energy side of the $(d, p),(d, n)$ and $(d, t)$ excitation functions [1-3,6,7], have been performed in the frame of the CRC formalism by using the code FRESCO [24] with the post form distorted-wave transition amplitude and finite-range interaction. The $n-p$ interaction in deuteron [16] as well as $d-n$ 

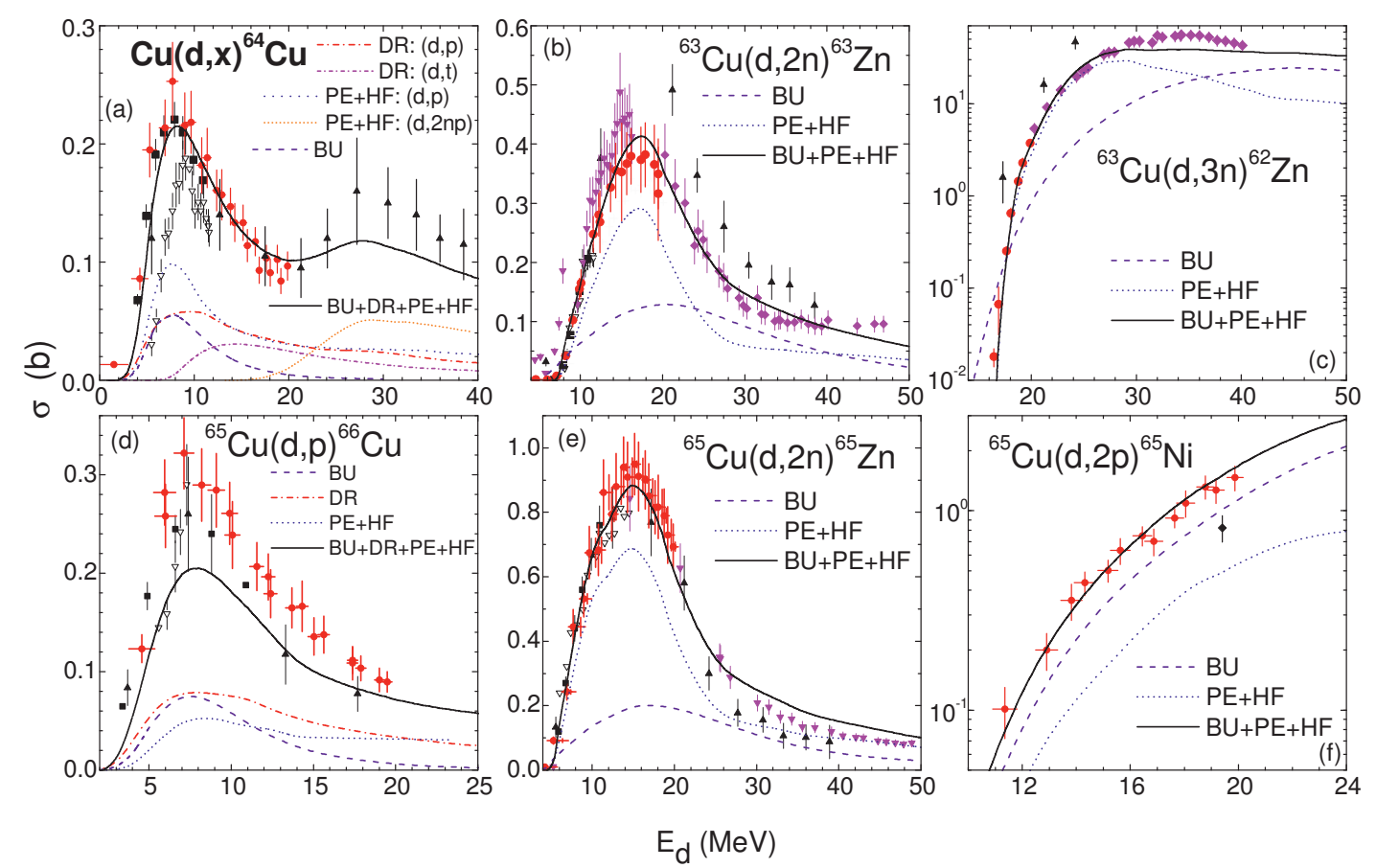

Fig. 3. (Color online) Comparison of measured data (see [6] and references therein) and complete analysis results (solid curves) taking into account the deuteron inelastic breakup (dashed), DR (dash-dotted for $(d, p)$ reactions and short dash-dotted for $(d, t)$ reaction), and $\mathrm{PE}+\mathrm{CN}$ (dotted and short-dotted for $(d, 2 n p)$ reaction) mechanism contributions to the deuteron interactions with ${ }^{n a t, 63,65} \mathrm{Cu}$ target nuclei [6].

interaction in triton [25] are assumed to have a Gaussian shape, while the transferred nucleon bound states were generated in a Woods-Saxon real potential.

Actually, the one-nucleon transfer reactions have been of critical importance for the nuclear structure studies. Thus, the spectroscopic factors extracted from the analysis of experimental angular distributions of the corresponding emitted particles did contribute to the validation of the nuclear shell model. Consequently, the rich systematics of the achieved experimental spectroscopic factors makes possible the calculation of almost total stripping and pick-up cross-section contribution to the deuteron activation.

A particular note should concern the $(d, t)$ pick-up mechanism contribution to the total $(d, t)$ activation cross section, usually neglected in spite of its essential contribution at low energies, namely at the energies between its threshold and the $(d, d n)$ and $(d, p 2 n)$ reaction thresholds that lead to the same residual nucleus.

\section{Statistical particle emission}

The PE or CN reaction mechanisms become important at the incident energies above the Coulomb barrier. We have calculated the corresponding reaction cross sections by means of the codes TALYS1.2 [26] and STAPRE-H [27], taking into account also the breakup and DR results discussed above. Particularly, a consistent local parameter set was involved within the detailed analysis carried out using the code STAPRE-H.

The local analysis results have obviously a higher accuracy, the consistent set of statistical model parameters being validated using independent experimental data for, e.g., neutron total cross sections, proton reaction cross sections, resonance data, and gamma-ray strength functions based on neutroncapture data $[2,6,28]$. On the other hand, no free parameter have been involved for the PE description 

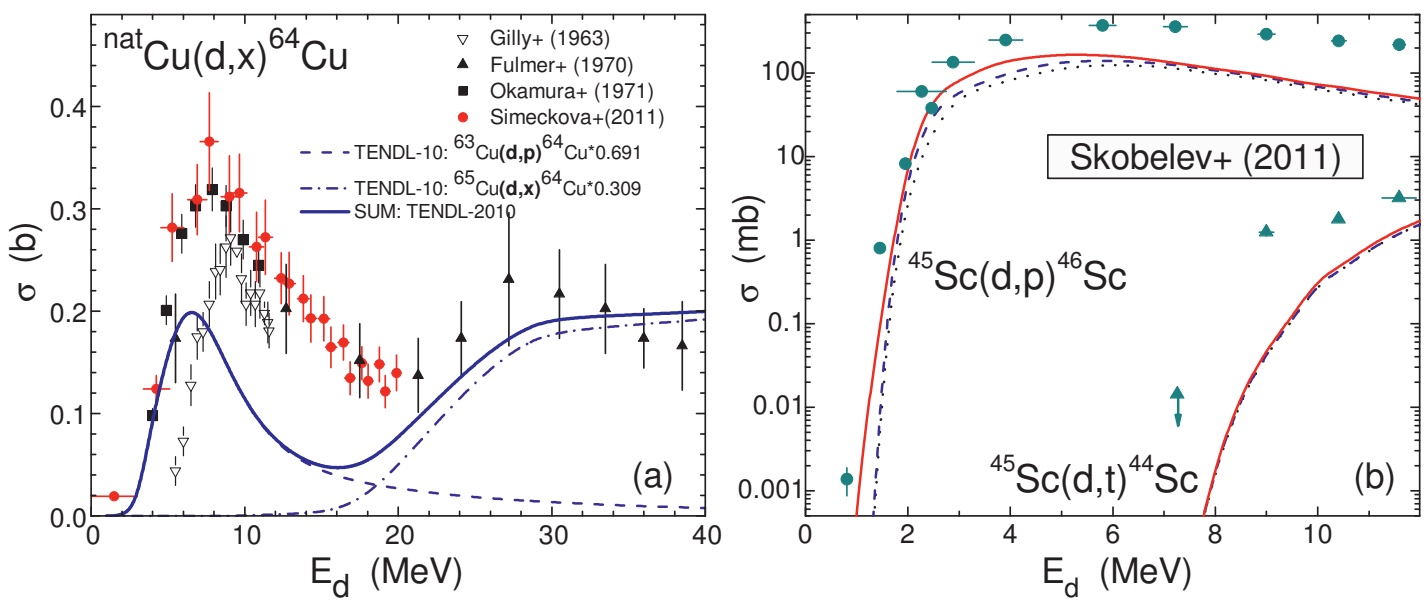

Fig. 4. (Color online) (a) Comparison of measured excitation function of the reaction ${ }^{n a t} \mathrm{Cu}(d, x){ }^{64} \mathrm{Cu}$ (see [6] and references therein) and the TENDL-2010 evaluated data [29] (solid curve) taking into account the contributions from both ${ }^{63} \mathrm{Cu}$ ( dashed curve) and ${ }^{65} \mathrm{Cu}$ (dot-dashed curve) target nuclei to the ${ }^{64} \mathrm{Cu}$ residual nucleus population. (b) Comparison of measured excitation functions for the reactions ${ }^{45} \mathrm{Sc}(d, p){ }^{46} \mathrm{Sc}$ (dots) and ${ }^{45} \mathrm{Sc}(d, t){ }^{44} \mathrm{Sc}$ (triangles) [30], and the calculated results obtained by using the code TALYS-1.2 [26] with default input parameters (dotted curves) as well as adjusted [30] OMP volume radius for deuterons (dashed) as well as for deuterons and protons (solid).

within the corresponding generalized Geometry Dependent Hybrid model. However a particular comment concerns the initial configuration of excited particles $(p)$ and holes $(h)$ for deuteron-induced reactions. Our previous analysis $[2,3,6]$ pointed out $2 p-1 h$ initial configuration instead of the more usual $3 p-1 h$. This point should be completely settled by further analysis of the measured and calculated cross sections obtained using various $(p, h)$ configurations .

As an example of complete analysis of deuteron interactions with nuclei in Fig. 3 is shown the comparison of the measured and calculated activation cross sections of ${ }^{n a t, 63,65} \mathrm{Cu}+d$ [6], all deuteroninduced reactions being properly described by the local consistent parameter set within the $\mathrm{PE}+\mathrm{CN}$ code STAPRE-H and taking into account also the breakup and DR contributions. These results validate the correctness of nuclear mechanism description that have been considered for the deuteron-nucleus interactions.

\section{Breakup and one-nucleon transfer reactions importance}

The importance of the breakup and one-nucleon transfer reaction mechanisms for the consistent analysis of the deuteron interactions with nuclei is revealed by the comparison of the experimental data and model calculations. Missing or unappropriate treatment of some reaction mechanism contributions lead to less satisfactory description of the corresponding experimental data. We may note in this respect the underestimation of measured excitation functions of the reaction ${ }^{n a t} \mathrm{Cu}(d, x){ }^{64} \mathrm{Cu}$ by the most recent TENDL-2010 evaluated data [29], based on TALYS code [26] [Fig. 4(a)]. The comparison between the Figs. 3(a) and 4(a) shows that the description of this excitation function can not be obtained by consideration of only the PE and $\mathrm{CN}$ reaction mechanisms by TALYS. Its underestimation by the TENDEL-2010 evaluation points out the important role of both the inelastic breakup enhancement through the ${ }^{63} \mathrm{Cu}(n, \gamma)$ capture reaction and the stripping ${ }^{63} \mathrm{Cu}(d, p)$ and pick-up ${ }^{65} \mathrm{Cu}(d, t)$ reactions.

\subsection{Stripping and pick-up neglection effects}

The spectroscopic studies performed by means of $(d, p)$ reactions have pointed out a maximum of the stripping $(d, p)$ excitation function around the deuteron incident energy of 8-10 MeV. Therefore 


\section{$\mathrm{CNR} * 11$}

the consistent analysis of $(d, p)$ processes at low incident energies has to include this mechanism on the basis of an appropriate theoretical frame as, e.g., the CRC method. On the other hand, omission of the transfer reaction contributions to the deuteron activation cross sections can be hidden by inadequate values adopted for various model parameters. Unfortunately, this seems to be the case of a recent analysis of deuteron interactions concerning the excitation functions of the ${ }^{45} \mathrm{Sc}(d, p){ }^{46} \mathrm{Sc}$ and ${ }^{45} \mathrm{Sc}(d, t){ }^{44} \mathrm{Sc}$ reactions [30]. It has considered the PE and CN processes by using the codes TALYS1.2 [26] and EMPIRE [31], but ignoring the stripping $(d, p)$ and pick-up $(d, t)$ contributions. The good description of the ${ }^{45} \mathrm{Sc}(d, p){ }^{46} \mathrm{Sc}$ excitation function reported by Skobelev et al. [30] in such conditions has determined us to reanalyze these reactions [32].

We have used in this respect the whole TALYS-1.2 default input parameter set and, additionally, the adjusted value $r_{v a d j}=1.12$ adopted in Ref. [30] for the reduced radius of the optical model (OM) volume potentials. The adjustment has been applied to both deuteron and proton OM potentials since no definite notice has been given in Ref. [30] on which it should be applied. The corresponding effects led together to an increase of the $(d, p)$ reaction cross section from $\sim 40 \%$ around the Coulomb barrier to less than $20 \%$ at $11.7 \mathrm{MeV}$. However, even these larger values underestimate the experimental data by a factor ranging from 2 to more than 4 at the same energies. Especially above the Coulomb barrier they are rather close to the content of the TENDL-2010 library [29]. Conversely, these evaluated data as well as those we have calculated are smaller by a factor $\sim 2$ with respect to the similar curve shown in Fig. 2 of Ref. [30]. As a result, the agreement with experimental data, e.g. the calculated ${ }^{45} \mathrm{Sc}(\mathrm{d}, \mathrm{p}){ }^{46} \mathrm{Sc}$ excitation function reported in Ref. [30], can not be replicated.

Nevertheless, the condition of a consistent input parameter set could be fulfilled only if the adopted change is validated by an OM analysis of elastic scattering data (e.g., Refs. $[1,2,6,7]$ ) or at least of total reaction cross sections $\sigma_{R}$ below the Coulomb barrier. However, this has not been the case of the above-mentioned OM reduced-radius adjustment by Skobelev et al. Moreover, a systematic analysis of the proton $\sigma_{R}$ in the same mass region showed that the default proton OM potential of TALYS does not underestimate this quantity but eventually overestimates it [28].

As a matter of fact the underestimation of the ${ }^{45} \mathrm{Sc}(d, p){ }^{46} \mathrm{Sc}$ experimental data by $\mathrm{PE}$ and $\mathrm{CN}$ calculations, using the TALYS or EMPIRE codes, could be really expected due to the related absence of a suitable consideration of the direct stripping mechanism. This assumption is experimentally endorsed by population of more than 80 discrete levels up to $\sim 4 \mathrm{MeV}$ excitation energy in ${ }^{46} \mathrm{Sc}$ [35], by $7 \mathrm{MeV}$ deuterons [33], as well as of $\sim 200$ discrete levels up to $\sim 7 \mathrm{MeV}$ excitation energy by 12 $\mathrm{MeV}$ deuterons [34]. Therefore a strong direct stripping contribution is maybe hidden by PE and $\mathrm{CN}$ parameters alteration by Skobelev et al. in Ref. [30].

The results that we have obtained through a similar analysis of the ${ }^{45} \mathrm{Sc}(\mathrm{d}, \mathrm{t}){ }^{44} \mathrm{Sc}$ reaction, at energies where the sensitivity to OM potential radius is much lower, is also shown in Fig. 4(b). These results are less than half of the measured data but rather identical with the TENDL-2010 data. However, the underestimation of the $(d, t)$ data by TALYS or EMPIRE calculations is due to the omission of the direct processes contribution, that is proved by spectroscopic studies of the ${ }^{44} \mathrm{Sc}$ discrete levels strongly populated through $(d, t)$ pick-up [36]. Actually, the lowest energy side of a $(d, t)$ excitation function, between its threshold and those of the $(d, d n)$ and $(d, p 2 n)$, can be described exclusively by the pick-up reaction mechanism as it is shown in Fig. 2. Nevertheless, the additional consideration of this reaction is consistent with the unitary analysis of nuclear model predictions taking into account all available data for various reaction channels (e.g., $[2,3,6-8,15])$. On the other hand, the evaluated [29] and presently calculated data are larger by a factor of $\sim 2$ with respect to the TALYS results of Skobelev et al.. Therefore, we could not replicate these results either. The latest additional reduction of their $(d, t)$ cross sections values might be a consequence of the stronger enhancement by means of $\mathrm{PE}$ and $\mathrm{CN}$ mechanisms of the $(d, p)$ reaction channel in Ref. [30].

\subsection{Breakup dominance versus fission}

A special point concerns the deuteron interactions with heavy nuclei, at energies around Coulomb barrier. Beyond the differences among the two breakup parameterizations $[1,14]$ discussed in Sec. 2, both of them point out the dominance of the breakup mechanism at the deuteron incident energies below and around the Coulomb barrier, as shown in Fig. 5(a) for deuteron interaction with ${ }^{231} \mathrm{~Pa}$. 

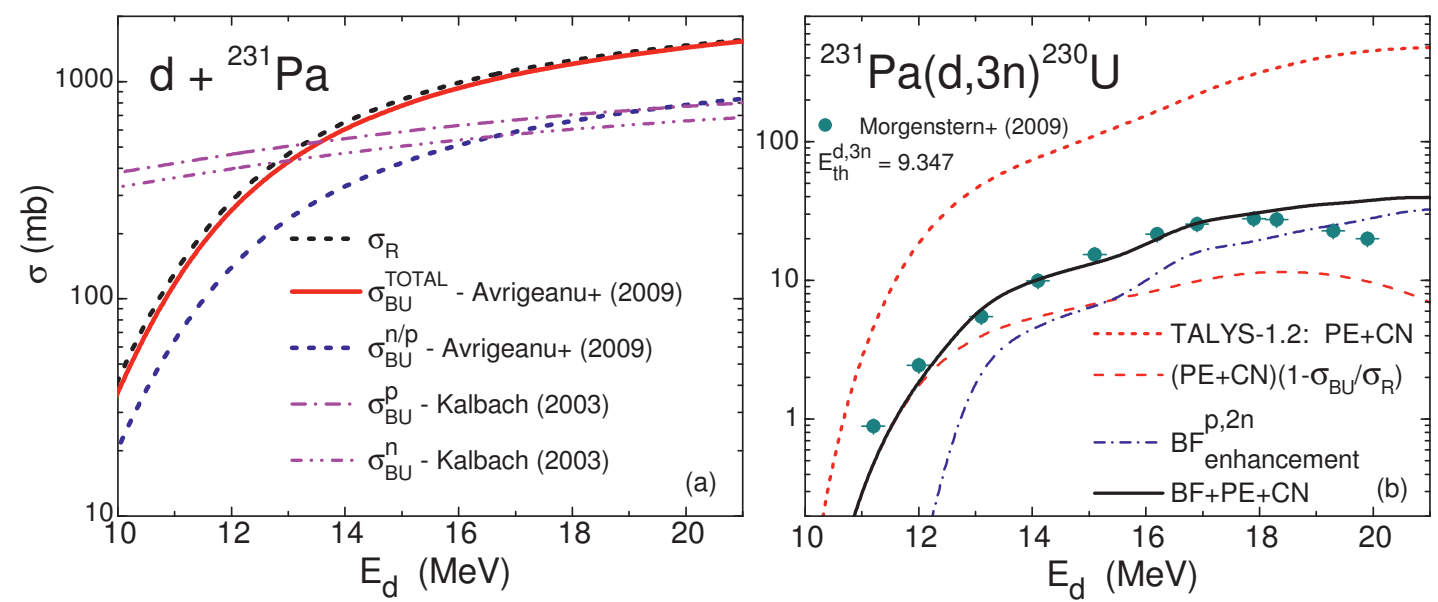

Fig. 5. (Color online) (a) Energy dependence of the total breakup (solid curve) and total nucleon-emission breakup cross sections [1] (dashed), and total proton- (dash-dotted curve) and neutron-emission breakup cross sections (dash-dot-dotted) of Ref. [14], for deuteron interactions with ${ }^{231} \mathrm{~Pa}$ around the Coulomb barrier. The related deuteron total reaction cross sections [26] are also shown (dotted). (b) Comparison of the measured excitation function of the reaction ${ }^{231} \mathrm{~Pa}(d, 3 n){ }^{230} \mathrm{U}$ [37] and the calculated $\mathrm{PE}$ and $\mathrm{CN}$ contributions without the breakup option in TALYS-1.2 (dotted), and including the correction for breakup leakage of incident deuteron flux (dashed), the inelastic-breakup (BF) enhancement (dash-dotted curve), and the sum of the three reaction mechanisms (solid).

On the other hand, a definite dominance but of the fission decay channel has been found by Morgenstern et al. [37] within the former ${ }^{231} \mathrm{~Pa}(d, 3 n){ }^{230} \mathrm{U}$ reaction cross-section analysis around the Coulomb barrier. They also noted that there is a significant decrease of the available compound-nucleus cross section due to the deuteron breakup, but with no related quantitative assessment. However, their fission cross sections obtained within the EMPIRE-2 computer code assumptions [31] are quite close to the deuteron total reaction cross sections (Fig. 3 of Ref. [37]). Nevertheless, since there are unfortunately no measured data in this respect, it is difficult to understand the quite large difference between the $(d, f)$ reaction cross sections predicted by them and the much lower calculated values either obtained using the code TALYS-1.2 [26] or provided by the TENDL-2010 library [29].

Concerning the breakup mechanism dominance, it results that further calculations of deuteron activation cross sections have to take into account both the huge leakage of initial flux toward the breakup process, as well as the inelastic breakup enhancement brought by the breakup nucleon interactions with the target nucleus. These opposite-effect contributions of the breakup mechanism are shown in Fig. 5(b) for the ${ }^{231} \mathrm{~Pa}(d, 3 n)^{230} \mathrm{U}$ reaction. The PE and $\mathrm{CN}$ contributions have been calculated by means of default input of TALYS-1.2 but without the breakup option [Fig. 5(b)]. This choice has been motivated by the omission of inelastic breakup enhancement consideration in the TALYS code, that is actually an open question of the codes for nuclear reaction cross sections calculations. Next, the breakup overall effect has been taken into account through a reduction factor $\left(1-\sigma_{B U} / \sigma_{R}\right)$, i.e. by subtracting the total breakup cross section $\sigma_{B U}$ [1] from the deuteron total reaction cross section $\sigma_{R}$. Following this reduction, the calculated $(d, 3 n)$ reaction cross section becomes, from largely overestimated, underestimated by a factor up to 3 above $E_{d} \sim 12 \mathrm{MeV}$ [Fig. 5 (b)]. Secondly, the inelastic breakup process expected to bring significant contributions to various reaction channels [2-7] is responsible for the enhancement of the ${ }^{230} \mathrm{U}$ production cross sections through the ${ }^{231} \mathrm{~Pa}(\mathrm{p}, 2 \mathrm{n}){ }^{230} \mathrm{U}$ process.

In order to calculate this breakup enhancement, the nucleon inelastic-breakup cross section $\sigma_{B F}^{n}$ have been multiplied by the ratio $\sigma_{(p, 2 n)} / \sigma_{(p, R)}$ corresponding to the weight of the above-mentioned reaction induced by the breakup protons on the ${ }^{231} \mathrm{~Pa}$ target nucleus $[2,6]$. We have used in this respect the measured ${ }^{231} \mathrm{~Pa}(p, 2 n)^{230} \mathrm{U}$ reaction cross sections [38], reported by the same team who measured also ${ }^{231} \mathrm{~Pa}(\mathrm{~d}, 3 \mathrm{n}){ }^{230} \mathrm{U}$ excitation function, while the proton total reaction cross section $\sigma_{R}$ has been 


\section{$\mathrm{CNR} * 11$}

calculated with TALYS-1.2. The ratio $\sigma_{(p, 2 n)} / \sigma_{(p, R)}$ has been expressed as a function of the deuteron incident energy, using the Kalbach [14] formula for the center-of-mass system centroid of the breakup protons energies. Since the breakup protons energy range of $\sim 9-14 \mathrm{MeV}$ corresponds to the incident energies of 11-20 MeV of the measured $(d, 3 n)$ excitation function [38], it is almost covered by the $(p, 2 n)$ reaction cross-sections measured between 10.6-23.8 MeV [38]. It is why the simultaneously analysis of $(p, 2 n)$ [38] and $(d, 3 n)$ [37] experimental excitation functions is the most powerful way to check the appropriateness of the empirical parameterization of the breakup components as well as the reaction mechanisms considered for the deuteron interactions with nuclei. The corresponding inelastic-breakup enhancement of the ${ }^{231} \mathrm{~Pa}(d, 3 n){ }^{230} \mathrm{U}$ activation cross section, shown in Fig. 5(b), goes thus from much lower values with respect to the $\mathrm{PE}+\mathrm{CN}$ contributions, at lowest energies, to even twice larger values above the Coulomb barrier.

Finally the suitable agreement of the sum of all three mechanism cross sections with the measured data [Fig. 5(b)] validates the correctness of the present approach. Of course there is a room for improving this agreement by an adequate search of the best parameterization for the formalisms associated to the PE and $\mathrm{CN}$ respectively, keeping in mind also the still significant differences between the fission predictions within various assumptions [37,26,29]. However, remembering that the deuteron breakup is the dominant process in this case, the attention should be focused on the breakup parametrization improvement. In this respect, for the case of a consistent consideration of the deuteron breakup effects, there are really needed complementary experimental studies like $(d, 3 n)$ and $(p, 2 n)$ reaction cross sections for the same target nucleus and within correlated incident-energy ranges.

\section{Conclusions}

The complexity of the deuteron interaction with nuclei has triggered the need to consider carefully several distinct reaction types, for which various approximations have been still widely-used so far. Therefore there are still notable deuteron-induced reaction studies that took into account only the statistical emission and eventually a 'reduction factor' of the compound nucleus cross section due to 'direct processes'. However, this reduction factor does not allow the distinction between processes such as the breakup and stripping mechanisms that lead to quite different energy ranges of the consequently emitted particles, the inelastic breakup enhancement to various reaction channels being totally ignored too. On the other hand, only the appropriate consideration of the DR makes possible the description of the low incident energies side of the excitation functions corresponding to channels which include stripping or pick-up mechanisms in addition to $\mathrm{PE}+\mathrm{CN}$ mechanisms.

It results the motivation of detailed theoretical treatment of each reaction mechanism contribution in order to obtain a reliable understanding of the interaction process as well as accurate values for the calculated deuteron activation cross sections.

Finally, the improvement of the deuteron breakup effects estimations requests complementary experimental studies of neutrons and protons induced reactions on the common target in respect with deuteron interaction processes and within correlated incident-energy ranges.

\section{Acknowledgments}

Work partly supported by the CNCS project No. PN-II-ID-PCE-2011-3-0450 and the ANCS project No. PN-09-37-01-05.

\section{References}

1. M. Avrigeanu, W. von Oertzen, R. A. Forrest, A. C. Obreja, F. L. Roman, V. Avrigeanu, Fusion Eng. Design, 84, (2009) 418.

2. P. Bém, E. Šimečková, M. Honusek, U. Fischer, S. P. Simakov, R. A. Forrest, M. Avrigeanu, A. C. Obreja, F. L. Roman, V. Avrigeanu, Phys. Rev. C 79, (2009) 044610. 
3. M. Avrigeanu, V. Avrigeanu, EPJ Web of Conferences 2, (2010) 01004.

4. M. Avrigeanu, V. Avrigeanu, J. Phys: Conf. Ser. 205, 012014 (2010).

5. E. Šimečková, P. Bém, M. Götz, M. Honusek, J. Mrázek, J. Novák, M. Štefánik, L. Závorka, M. Avrigeanu, V. Avrigeanu, EPJ Web of Conferences 8, (2010) 07002.

6. E. Šimečková, P. Bém, M. Honusek, M. Štefánik, U. Fischer, S. P. Simakov, R. A. Forrest, A.J. Koning, J.-C. Shublet, M. Avrigeanu, F. L. Roman, V. Avrigeanu, Phys. Rev. C 84, (2011) 014605.

7. M. Avrigeanu, V. Avrigeanu, J. Korean Phys.Soc. 59, (2011) 903.

8. M. Avrigeanu, W. von Oertzen, U. Fischer, V. Avrigeanu, Nucl. Phys. A 759, (2005) 327.

9. J. Pampus, J. Bisplinghoff, J. Ernst, T. Mayer-Kuckuk, J. Rama Rao, G. Baur, F. Rosel, D. Trautmann, Nucl. Phys. A 311, (1978) 141.

10. J. R. Wu, C. C. Chang, H. D. Holmgren, Phys. Rev. C 19, (1979) 370.

11. J. Kleinfeller, J. Bisplinghoff, J. Ernst, T. Mayer-Kuckuk, G. Baur, B. Hoffmann, R. Shyam, F. Rosel, D. Trautmann, Nucl. Phys. A 370, (1981) 205.

12. N. Matsuoka, M. Kondo, A. Shimizu, T. Saito, S. Nagamachi, H. Sakaguchi, A. Goto, F. Ohtani, Nucl. Phys. A 345, (1980) 1.

13. M. G. Mustafa, T. Tamura, T. Udagawa, Phys. Rev. C 35, (1987) 2077.

14. C. Kalbach Walker, TUNL Progress Report XLII (2002-2003) p. 82-83, Triangle University Nuclear Laboratory; www.tunl.duke.edu/publications/tunlprogress/2003/ .

15. M. Avrigeanu, A. M. Moro, Phys. Rev. C 82, (2010) 037601.

16. M. Kawai, M. Kamimura, K. Takesako, Prog. Theor. Phys. Suppl. 184, (1969) 118.

17. N. Austern, Y. Iseri, M. Kamimura, M. Kawai, G. Rawitscher, M. Yahiro, Phys. Rep. 154, (1987) 125.

18. R. A. D. Piyadasa, M. Kawai, M. Kamimura, M. Yahiro, Phys. Rev. C 60, (1999) 044611.

19. A. M. Moro, F. M. Nunes, Nucl. Phys. A 767, (2006) 138.

20. A. M. Moro, J. M. Arias, J. Gómez-Camacho, F. Pérez-Bernal, Phys. Rev. C 80, (2009) 054605.

21. A. M. Moro, F. M. Nunes, R. C. Johnson, Phys. Rev. C 80, (2009) 064606.

22. C. Kalbach Walker, in: TUNL Progress Report, vol. XLVI (2007), p. 78. www.tunl.duke.edu/publications/tunlprogress/2007/tunlxlvi.pdf .

23. Experimental Nuclear Reaction Data (EXFOR), www-nds.iaea.or.at/exfor; R.Michel, M.Galas, Appl. Rad. Isotopes 34, (1983) 1325, EXFOR A0209 entry; F. Ditroi, F. Tarkanyi, S. Takacs, A. Hermanne, M. Baba, A.V. Ignatyuk, Nucl. Instr. Meth. B 268, (2010) 2571, EXFOR D4232 entry; F. Tarkanyi, A. Hermanne, F. Ditroi, S. Takacs, Nucl. Instr. Meth. B 255,(2007) 297, EXFOR D4156 entry.

24. I. J. Thompson, Comput. Phys. Rep. 7, (1988) 167; v. FRES 2.3 (2007).

25. P. Guazzoni, L. Zetta, A. Covello, A. Gargano, B.F. Bayman, T. Faestermann, G. Graw, R. Hertenberger, H.-F. Wirth, M. Jaskola, Phys. Rev. C 83, (2011) 044614.

26. A. J. Koning, S. Hilaire, M. C. Duijvestijn, TALYS-1.0, in Proc. Int. Conf. on Nuclear Data for Science Technology, Nice, 2007 (EDP Sciences, Paris, 2008), p. 211; version TALYS-1.2, December 2009; http://www.talys.eu/home/ .

27. M. Avrigeanu, V. Avrigeanu, IPNE Report NP-86-1995, Bucharest, 1995, Refs. therein; News NEA Data Bank 17,(1995) 22.

28. M. Avrigeanu, S. Chuvaev, A. A. Filatenkov, R. A. Forrest, M. Herman, A. J. Koning, A. J. M. Plompen, F. L. Roman, V. Avrigeanu, Nucl. Phys. A 806, (2008) 15.

29. A.J Koning, D. Rochman, TENDL-2010: TALYS-Based Evaluated Nuclear Data Library (December 8, 2010), http://www.talys.eu/tendl-2010/

30. N.K. Skobelev, A.A. Kulko, V. Kroha, V. Burjan, Z. Hons, A.V. Daniel, N.A. Demeskhina, R. Kalpakchieva, A. Kugler, J. Mrazek, Yu.E. Penoinzhkevich, S. Piskor, E. Simeckova, E.I. Voskoboynik, J. Phys. G: Nucl. Part. Phys. 38, (2011) 035106.

31. M. Herman, R. Capote, B.V. Carlson, P. Oblozinsky, M. Sin, A. Trkov, H. Wiencke, V. Zerkin, Nucl. Data Sheets 108, (2007) 2655.

32. M. Avrigeanu, V. Avrigeanu, http://arxiv.org/abs/1105.1881v1.

33. R. Rapaport, A. Sperduto, W.W. Bruechner, Phys. Rev. 151, (1966) 939.

34. J.N. Roy, A.R. Majunder, H.M. Sen Gupta, Phys. Rev. C 46, (1992) 144. 


\section{CNR*11}

35. S. -C. Wu, Nucl. Data Sheets 91, (2000) 1.

36. J.A. Cameron, Singh Balraj, Nucl. Data Sheets 88, (1999) 299.

37. A. Morgenstern, O. Lebeda, J. Stursa, R. Capote, M. Sin, F. Bruchertseifer, B. Zielinska, C. Apostolidis, Phys. Rev. C 80, (2009) 054612.

38. A. Morgenstern, O. Lebeda, J. Stursa, F. Bruchertseifer, R. Capote, J. McGinley, G. Rasmussen, M. Sin, B. Zielinska, C. Apostolidis, Anal. Chem. 80, (2008) 8763; Experimental Nuclear Reaction Data (EXFOR); www-nds.iaea.or.at/exfor; A.Morgenstern et al., EXFOR D0562 entry. 